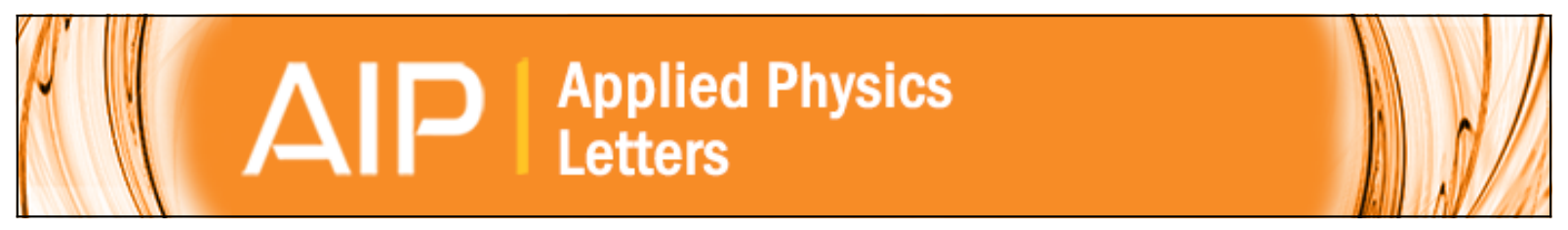

\title{
Photonic pseudo-gap-based modification of photoluminescence from CdS nanocrystal satellites around polymer microspheres in a photonic crystal
}

Y. Lin, J. Zhang, E. H. Sargent, and E. Kumacheva

Citation: Applied Physics Letters 81, 3134 (2002); doi: 10.1063/1.1515881

View online: http://dx.doi.org/10.1063/1.1515881

View Table of Contents: http://scitation.aip.org/content/aip/journal/apl/81/17?ver=pdfcov

Published by the AIP Publishing

\section{Articles you may be interested in}

Surface states effect on photoluminescence of CdS colloidal nanocrystals

J. Appl. Phys. 113, 184306 (2013); 10.1063/1.4804255

Modification of emission of CdTe nanocrystals by the local field of Langmuir-Blodgett colloidal photonic crystals J. Appl. Phys. 104, 103118 (2008); 10.1063/1.2981087

Sharp photoluminescence of CdSeS nanocrystals embedded in silica glass

Appl. Phys. Lett. 82, 49 (2003); 10.1063/1.1526173

Photoluminescence of ZnS:Mn embedded in three-dimensional photonic crystals of submicron polymer spheres Appl. Phys. Lett. 76, 3513 (2000); 10.1063/1.126691

CdS photoluminescence inhibition by a photonic structure

Appl. Phys. Lett. 73, 1781 (1998); 10.1063/1.122280

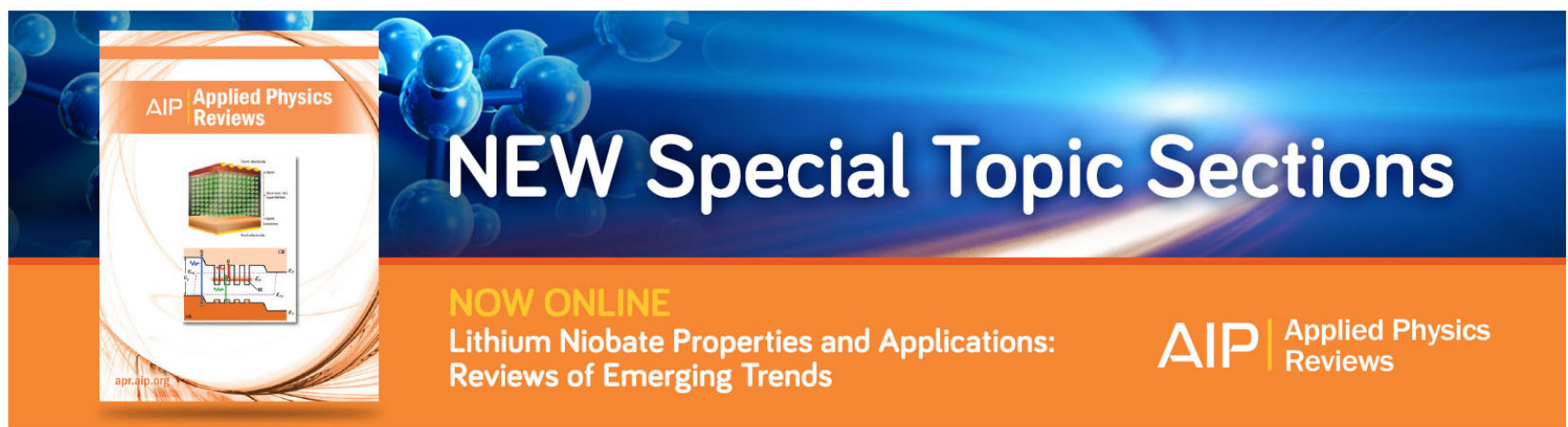




\title{
Photonic pseudo-gap-based modification of photoluminescence from CdS nanocrystal satellites around polymer microspheres in a photonic crystal
}

\author{
Y. Lin ${ }^{\mathrm{a})}$ \\ Department of Electrical and Computer Engineering, University of Toronto, Ontario M5S 3G4, Canada \\ J. Zhang \\ Department of Chemistry, University of Toronto, Ontario M5S 3H6, Canada \\ E. H. Sargent \\ Department of Electrical and Computer Engineering, University of Toronto, Ontario M5S 3G4, Canada \\ E. Kumacheva \\ Department of Chemistry, University of Toronto, Ontario M5S 3H6, Canada
}

(Received 5 July 2002; accepted 28 August 2002)

\begin{abstract}
We report the combination of microsphere self-organization to form a photonic crystal, providing spectrally and angularly dependent electromagnetic structural resonances; with nanocrystal growth in situ on microsphere surfaces, providing optical functionalization with spectral control achieved through the quantum size effect. We demonstrate this material system using CdS nanocrystals coating the surfaces of poly(methyl methacrylate)-poly(methacrylic acid) (PMMA-PMAA) micrometer spheres. The in situ synthesis of the CdS nanocrystals on the surface of the PMMA/ PMAA microspheres preserves the propensity of the hybrid microspheres to form ordered colloid arrays. Luminescence from surface states ensures that light is emitted at energies significantly below the absorption edge of the emitting species. Transmission and photoluminescence measurements reveal the interaction of the photonic stop band with photoluminescence from the nanocrystals.

(C) 2002 American Institute of Physics. [DOI: 10.1063/1.1515881]
\end{abstract}

Photonic crystals-materials whose dielectric constant exhibits a periodic modulation-inhibit electromagnetic wave propagation over a band of frequencies for a particular polarization and propagation direction relative to the crystal axes. Photonic band-gap materials may enable compact optical integrated circuits via sharp-bend waveguides, lowthreshold laser arrays, and optical transistor action. ${ }^{1-4}$

Colloidal microspheres made up of $\mathrm{SiO}_{2}$ or polymers may, under suitable conditions, self-organize into threedimensional periodic arrays, ${ }^{2,5-7}$ often adopting the facecentered-cubic lattice type. Recently, enhanced control has been demonstrated over the formation of such synthetic opals, with both the placement of the crystals and the nature of their ordering being controlled by substrate patterning. ${ }^{8}$

Active optical function may be achieved by introducing materials such as semiconductors into the voids between microspheres following photonic crystal formation. Nonlinear optical and light-emitting infiltrates are of particular interest. ${ }^{9-17}$

We present herein the optical behavior of material produced using a distinctive approach. Instead of infiltration, semiconductor nanocrystals were grown in situ on the surface of microspheres prior to their self-organization into the photonic crystal, achieving refined control over the quantum dot satellite orbiting each latex particle. Specifically, we report growth of CdS nanocrystals on the surface of poly(methyl methacrylate)-poly(methacrylic acid)

a)Electronic mail: yuankun.lin@utoronto.ca
(PMMA-PMAA) microspheres. The methods result in a high degree of control over the loading and the size of semiconductor nanocrystals within the photonic crystal. Surface functionalization, resulting in placement of the active species-semiconductor nanocrystals - at the interface between air and the dielectric, can be expected to provide comparable overlap with the active species of Bloch modes associated with the air band and the dielectric band.

We report herein optical studies that seek to uncover the interaction of a photonic stop band with photoluminescence from deep surface states of $\mathrm{CdS}$ nanocrystals at the dielectric-air interface. Luminescence from surface states ensures that light is emitted at energies significantly below the absorption edge of the emitting species.

The details of in situ synthesis of CdS-doped PMMAPMAA microspheres have been reported elsewhere. ${ }^{18}$ In summary, the surface carboxyl group of PMMA-PMAA microbeads was deprotonated at a $p \mathrm{H}=8.5$. Then, $\mathrm{Cd}^{2+}$ was added and attached onto the surface of the microbeads by virtue of the strong affinity between $\mathrm{Cd}^{2+}$ and the carboxyl group. Following this ion-exchange process, $\mathrm{Na}_{2} \mathrm{~S}$ was introduced to the dispersion. The color of the dispersion became yellow, indicating the formation of CdS nanocrystals. No stabilizer was needed. The average size of the CdS nanocrystals was measured, using transmission electron microscopy, to be about $6 \mathrm{~nm}^{18}$

Optical absorption of the monodispersed colloidal suspension of CdS-nanocrystal-coated PMMA-PMAA microspheres was measured using a Cary 500 spectrophotometer. Photoluminescence (PL) measurements were acquired using 


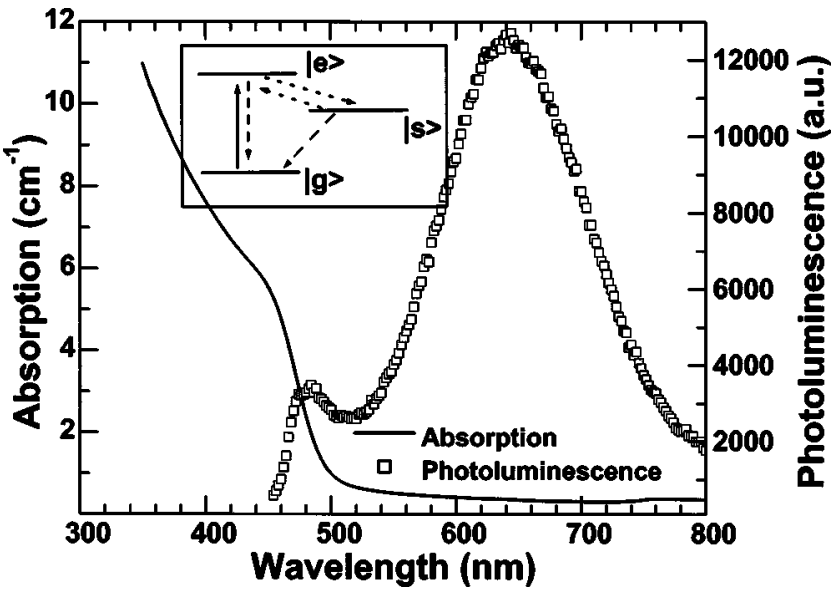

FIG. 1. Optical absorption from aqueous dispersions of CdS/PMMA/PMAA hybrid spheres diluted in dimethyl sulfoxide (DMSO) with DMSO as a reference, together with photoluminescence (PL) spectrum from colloidal CdS/PMMA/PMAA spheres. The narrow peak is a band-edge emission from the radiative decay from excited state $|e\rangle$ to ground state $|g\rangle$ while the broad peak is a radiative decay of the carrier captured by nanocrystal surface trap state $|s\rangle$ from the excited state, as shown in the inset.

a PTI spectrofluorometer excited using a $\mathrm{HeCd}$ laser operating at $442 \mathrm{~nm}$.

Figure 1 shows the absorption and luminescence spectra of PMMA/PMAA hybrid spheres coated with aqueous CdS. To isolate the effect of absorption alone, rather than scattering, the samples measured in Fig. 1 were diluted in the dimethyl sulfoxide (DMSO) (refractive index $n=1.479$ ) to achieve refractive index matching ( $n=1.48$ for PMMA/ PMAA). The onset absorption at $500 \mathrm{~nm}$ suggests a nanocrystal size of slightly under $6 \mathrm{~nm} .{ }^{19}$ The luminescence spectrum displays band-edge emission at the wavelength close to the onset of absorption $(\sim 480 \mathrm{~nm})$; plus an additional strong broad peak accompanied by minimal absorption (560-740 $\mathrm{nm})$ as depicted in the three-level diagram in the inset of Fig. 1. The broad peak is attributed to the recombination of charge carriers in deep traps of surface localized states. ${ }^{19,20}$ The PL spectra of the CdS nanocrystal are very similar to those prepared in other ways, ${ }^{19,20}$ including in glass. ${ }^{21}$

Photonic crystals were fabricated by casting the colloidal suspension onto a glass slide. An iridescent film with thickness of $0.5 \mathrm{~mm}$ was formed following evaporation of the solvent. The atomic force microscope image (measured with Digital Instrument scanning probe microscopy; tapping mode) of Fig. 2, depicting the top surface of the colloidal photonic crystal, reveals a well-ordered triangular arrangement corresponding to the (111) surface of a face-centeredcubic structure..$^{5-7}$ We estimate the sphere diameter to be $289 \pm 4 \mathrm{~nm}$.

We show in Fig. 3(a) the transmittance spectrum of the doped photonic crystals for a number of incidence angles with respect to the normal to the (111) surface. For $0^{\circ}$ incidence the spectrum shows a dip in the range 640-660 nm associated with the stop band arising from Bragg diffraction from (111) planes. The stop band is removed in the sample infiltrated with index-matching DMSO, providing evidence that the Bragg diffraction observed in transmission results from the period dielectric structure consisting of spheres. The spectral position of the center of the stop band shifts to shorter wavelengths as the incident beam angle is increased.

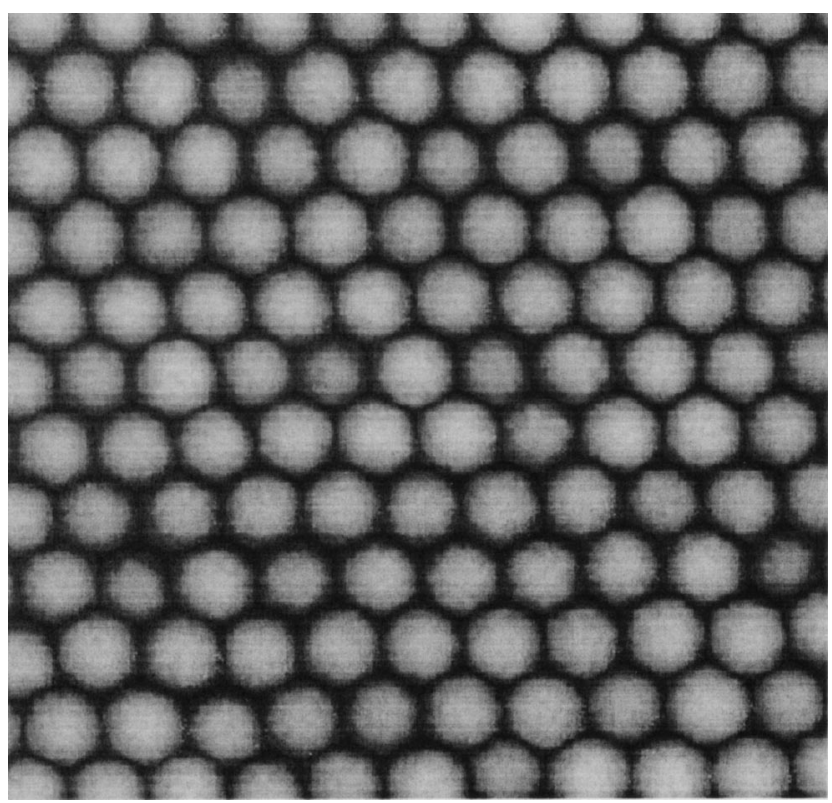

FIG. 2. Atomic force microscope (AFM) of a $3 \times 3 \mu \mathrm{m}^{2}$ region of the (111) surface of the photonic crystal made from 289-nm-diam spheres.

The data fit the Bragg law: $\lambda_{c}=1.632 d \sqrt{n_{\mathrm{eff}}^{(2)}-\sin ^{2} \theta}$, where $n_{\text {eff }}$ is the effective refractive index (fitting gives 1.387) and $d$ is the sphere diameter. ${ }^{5,9}$

We show in Fig. 3(b) photoluminescence spectra of the composite materials, also for a number of observation angles. Relative to the index-matched PL spectrum (top curve), the uninfiltrated material exhibits spectral dips in its PL spectrum, for example, at $650 \mathrm{~nm}$ for PL collected at $0^{\circ}$.

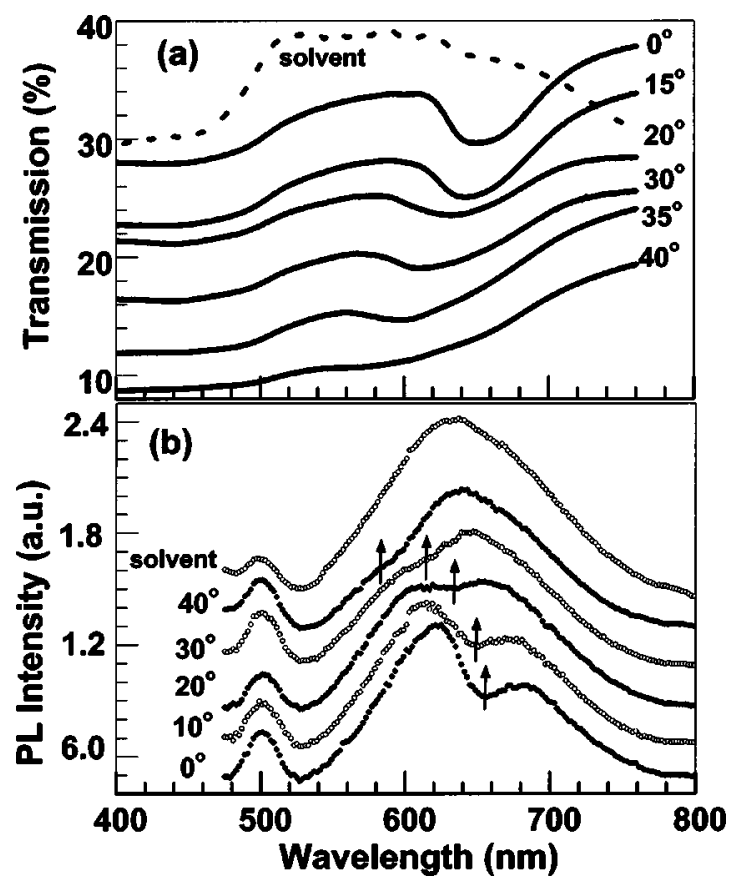

FIG. 3. (a) Optical transmission for different incidence angles with respect to the surface normal of photonic crystal doped with CdS nanocrystal together with the transmission from solvent DMSO infiltrated photonic crystal (dash line, shifted down). (b) PL spectra from the CdS nanocrystal doped in photonic crystal as a function of observation angles with respect to the (111) surface of the photonic crystal. The spectrum from the solvent DMSO infiltrated sample is also shown. The vertical arrows indicate the wavelength of the center of the stop band for the angle at which PL is acquired. 
We indicate in Fig. 3(b) with vertical arrows the wavelength of the center of the stop band for the angle at which PL is acquired. The luminescence dips are seen to occur at the center of the angular stop band in each case, due to the interaction of the photonic stop band and light emission.

While previous authors have reported ${ }^{9,11}$ the modification of bulk polycrystalline $\mathrm{CdS}$ photoluminescence in photonic crystals, we report herein incorporation into a selforganized photonic crystal a population of CdS nanocrystals. These preserve the effects of quantum confinement, such as discrete electronic states and optical nonlinearities tunable with the size of the nanocrystal. The nanocrystal-inphotonic-crystal system provides an attractive model system for the validation of recent theoretical work on two-level atomic systems inside full and partial photonic band gaps. ${ }^{4}$

In summary, we have shown the combination of microsphere self-organization to form a photonic crystal, providing electromagnetic structural resonances; with nanocrystal growth in situ on microsphere surfaces, providing optical functionalization with spectral control achieved through the quantum size effect. The in situ synthesis of the CdS nanocrystals on the surface of PMMA/PMAA microspheres is key, preserving the propensity of the hybrid microspheres to form ordered colloid arrays. Photonic crystals are designed and fabricated to study the interaction of a photonic stop band with photoluminescence from deep surface states of $\mathrm{CdS}$ nanocrystals at the dielectric-air interface. Luminescence from surface states ensures that light is emitted at energies significantly below the absorption edge of the emitting species. A dip in the emission has been observed in the material system at the center of the angular stop band, due to the interaction of the photonic pseudogap and phololuminescence.

One of the authors (Y.L.) thanks National Science and Engineering Research of Canada (NSERC) for a postdoctoral fellowship. The authors thank NSERC and Nortel Networks for support of the research.

${ }^{1}$ E. Yablonovitch, Phys. Rev. Lett. 58, 2059 (1987); S. John, ibid. 58, 2486 (1987).
${ }^{2}$ A. Blanco, E. Chomski, S. Grabtchak, M. Ibisate, S. John, S. W. Leonard, C. López, F. Meseguer, H. Míguez, J. P. Mondia, G. Ozin, O. Toader, and H. M. van Driel, Nature (London) 405, 437 (2000).

${ }^{3}$ A. Mekis, J. C. Chen, I. Kurland, S. Fan, P. R. Villeneuve, and J. D. Joannopoulos, Phys. Rev. Lett. 77, 3787 (1996).

${ }^{4}$ S. John and M. Florescu, J. Opt. A, Pure Appl. Opt., 3, S103 (2001).

${ }^{5}$ H. Míguez, C. López, F. Meseguer, A. Blanco, L. Vázquez, R. Mayoral, M. Ocaña, V. Fornés, and A. Mifsud, Appl. Phys. Lett. 71, 1148 (1997).

${ }^{6}$ Y. A. Vlasov, X. Bo, J. C. Sturm, and D. J. Norris, Nature (London) 414, 289 (2001).

${ }^{7}$ P. Jiang, J. F. Bertone, and V. L. Colvin, Science 291, 453 (2001).

${ }^{8}$ E. Kumacheva, R. K. Golding, M. Allard, and E. H. Sargent, Adv. Mater. 14, 221 (2002).

${ }^{9}$ A. Blanco, C. López, R. Mayoral, H. Míguez, F. Meseguer, A. Mifsud, and J. Herrero, Appl. Phys. Lett. 73, 1781 (1998); H. Míguez, A. Blanco, F. Meseguer, C. López, H. M. Yates, M. E. Pemble, V. Fornés, and A. Mifsud, Phys. Rev. B 59, 1563 (1999); A. Blanco, H. Miguez, F. Meseguer, C. López, F. López-Tejeira, J. Sánchez-Dehesa, Appl. Phys. Lett. 78, 3181 (2001).

${ }^{10}$ S. G. Romanov, A. V. Fokin, and R. M. De La Rue, Appl. Phys. Lett. 74, 1821 (1999); S. G. Romanov, A. V. Fokin, and R. M. De La Rue, ibid. 76, 1656 (2000); S. G. Romanov, T. Maka, C. M. Sotomayor Torres, M. Müller, and R. Zentel, ibid. 75, 1057 (1999).

${ }^{11}$ Yu. A. Vlasov, K. Luterova, I. Pelant, B. Hönerlage, and V. N. Astratov, Appl. Phys. Lett. 71, 1616 (1997).

${ }^{12}$ V. N. Astratov, A. M. Adawi, M. S. Skolnick, V. K. Tikhomirov, V. Lyubin, D. G. Lidzey, M. Ariu, and A. L. Reynolds, Appl. Phys. Lett. 78, 4094 (2001).

${ }^{13}$ J. Zhou, Y. Zhou, S. Buddhudu, S. L. Ng, Y. L. Lam, and C. H. Kam, Appl. Phys. Lett. 76, 3513 (2000).

${ }^{14}$ Y. A. Vlasov, N. Yao, and D. Norris, Adv. Mater. 11, 165 (1999); A. Rogach, A. Susha, F. Caruso, G. Sukhorukov, A. Kornowski, S. Kershaw, H. Möhwald, A. Eychmüller, and H. Weller, ibid. 12, 333 (2000).

${ }^{15}$ T. Yamasaki and T. Tsutsui, Appl. Phys. Lett. 72, 1957 (1998).

${ }^{16}$ H. P. Schriemer, H. M. van Driel, A. F. Koenderink, and W. L. Vos, Phys. Rev. A 63, 011801 (2001); A. F. Koenderink, L. Bechger, H. P. Schriemer, A. Lagendijk, and W. L. Vos, Phys. Rev. Lett. 88, 143903 (2002).

${ }^{17}$ S. V. Gaponeńko, A. M. Kapitonov, V. N. Bogomolov, A. V. Prokofiev, A. Eychmüller, and A. L. Rogach, JETP Lett. 68, 142 (1998); E. P. Petrov, V. N. Bogomolov, I. I. Kalosha, and S. V. Gaponenko, Phys. Rev. Lett. 81, 77 (1998).

${ }^{18}$ J. Zhang, N. Coombs, and E. Kumacheva, J. Am. Chem. Soc. (in press).

${ }^{19}$ L. Spanhel, M. Haase, H. Weller, and A. Henglein, J. Am. Chem. Soc. 109, 5649 (1987).

${ }^{20}$ M. G. Bawendi, P. J. Carroll, W. L. Wilson, and L. E. Brus, J. Phys. Chem. 96, 946 (1992).

${ }^{21}$ J. Butty, Y. Z. Hu, N. Peyghambarian, Y. H. Kao, and J. D. Mackenzie, Appl. Phys. Lett. 67, 2672 (1995); J. Butty, N. Peyghambarian, Y. H. Kao, and J. D. Mackenzie, ibid. 69, 3224 (1996). 